\section{DESIGN OF THE MM-WAVE SYSTEM OF THE ITER ECRH UPPER LAUNCHER}

A.G.A. Verhoeven, W.A. Bongers, A. Bruschi**, S. Cirant**, B.S.Q. Elzendoorn, G. Gantenbein***, M.F. Graswinckel, R. Heidinger*, W. Kasparek***, O.G. Kruyt, B. Lamers, B. Piosczyk*, B. Plaum***, D.M.S. Ronden, G. Saibene**** and H. Zohm*****

FOM-Institute for Plasma Physics Rijnhuizen, Association EURATOM-FOM, Nieuwegein, The Netherlands, ${ }^{\star} F Z K$, Karlsruhe, ${ }^{* *} \mathrm{CNR}$, Milan, ${ }^{* *}$ Univ Stuttgart, ${ }^{\star * \star \star} E F D A$, Garching, ${ }^{\star * \star \star *}$ Max-Planck, Garching e-mail: verhoeven@rijnh.nl www.rijnh.nl

\section{Introduction}

The aim of the mm-wave system to be installed in the ITER upper-port system is to inject Electron Cyclotron Waves (ECW) in the ITER plasma in order to stabilize neoclassical tearing modes (NTM). Each upper-port launcher consists of eight mm-wave lines capable of transmitting high power up to $2 \mathrm{MW}$ at 170 GHz. Within a broadly based EFDA collaboration conducting design and R\&D activities in preparation for the construction of an ITER upper port launcher, the FOM institute is leading the mm-wave design activity.

In order to exploit the capability of ECW for localized heating and current drive over a range of plasma radii in ITER, the ECH\&CD upper port launcher must have a beam steering capability. To avoid movable mirrors at the plasmafacing end of the launcher, the concept of remote mm-wave beam steering (RS) is used, having a corrugated square waveguide within the launcher and the steerable optic is then placed outside of the first confinement boundary of the vacuum vessel.

\section{System lay-out}

Starting from the gyrotrons, the mm-wave power will be transmitted towards the tokamak by circular evacuated waveguides with an aperture of $63.5 \mathrm{~mm}$. Steering of the beam over a range of $+/-12^{\circ}$ will be achieved by a mirror system consisting of a combination of curved and rotating mirrors. Via the mirror system the beam will be directed into a square corrugated waveguide of $44 \times 44 \mathrm{~mm}$ with a length of $4.4 \mathrm{~m}$. A single-disk diamond window and an isolation valve will provide the tritium boundary between the primary and secondary vacuum. At the end of the square waveguide, mm-wave beams will be guided through penetrations in the front-shield blanket module by a fixed-mirror system towards the ITER plasma. This mirror will have focusing properties in both directions. This will result in a smaller effective steering range in the plasma of $+/-6^{\circ}$ to $+/-12^{\circ}$. The latest 3-D CATIA model (June 2004) incorporates the newest requirements on steering range $\left(+/-10.5^{\circ}\right.$ for the lower row and $+/-8^{\circ}$ for the upper) and launching angles. This was based on the physics-based performance analysis that has been performed recently $[1,2,3,4,5]$.

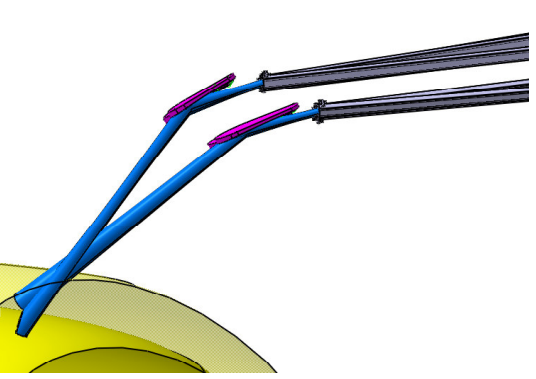

Figure 1: The two beams directed to the $\mathrm{q}=2$ surface.

\section{Design parameters}

Frequency:

Focusing last mirror:

Input scanning range:

Resulting scan upper row:

Resulting scan lower row: $\alpha$ :

Distance wg/mirror

$170 \mathrm{GHz}$

Both in toroidal and poloidal direction

$\pm 12^{\circ}$

$\pm 6.2^{\circ}$ real scan; $\pm 8.0^{\circ}$ from mirror midpoint $\pm 9.0^{\circ}$ real scan; $\pm 10.5^{\circ}$ from mirror midpoint upper row: $-61.3^{\circ}$; lower: $-50.0^{\circ}$

upper row: $-18^{\circ}$, lower: $-20^{\circ}$ upper row: $300 \mathrm{~mm}$; lower: $250 \mathrm{~mm}$

$\alpha \quad$ The poloidal launching angle: the angle between the poloidal component of the nominal beam centerline and the horizontal plane.

$\beta$ The toroidal launching angle: the angle between the nominal beam centerline and its poloidal component.

\section{Description of mm-wave system}

The 2-MW ECRH mm-wave system has to operate under various vacuum conditions. The transmission line is partly quasi optical, at the location of the remote steerable mirror and at the location of the plasma facing fixed mirror, see fig.2. As primary vacuum boundary a diamond window is placed after the steerable mirror in order to prevent tritium and beryllium contamination.

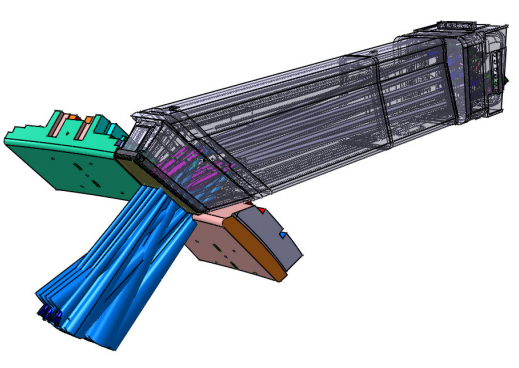

Figure 2: The reference model for full focusing

\begin{tabular}{llll}
\hline Ec-13 6/28/2004 & Toon Verhoeven & Page 1 of 3
\end{tabular} 
The resulting neutron load in the port-duct will be essential for the possibility of maintenance on the system after DT operation [6]. Behind the diamond window is an isolation valve and the entrance of the square corrugated waveguide.

\section{Mirror steering mechanism}

As it leaves the gyrotron, the microwave beam will be guided through a waveguide with a circular cross-section of $63.5 \mathrm{~mm}$. Steering of the beam will be achieved by a steerable mirror mechanism, placed at the end of the circular waveguide. The plane, in which steering takes place, depends on the scanning plane orientation of the outgoing beam. First, the beam will be focused by a fixed, curved mirror (M1) to limit the spot size at the beginning of the square waveguide. The steerable mirror (M2) makes a combined translating and rotating movement in the drawing plane in order to steer the beam through the waveguide aperture, see fig. 3 .

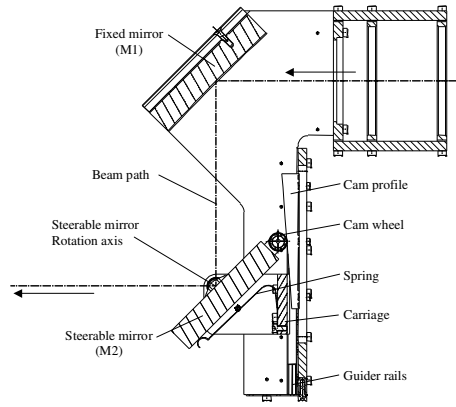

Figure 3: Mirror steering mechanism, cross section

The initial requirement is that the beam axis has to cross the waveguide axis always at the point of entry of the waveguide. The easiest way to achieve this is to let the mirror rotate around the mirror midpoint, located on the (flat) mirror's surface (axis perpendicular to the sketch surface), combined with a simultaneous translation along the incoming beam direction (vertical, as seen in figure 3 ). By using a cam-mechanism, only one actuator is needed to perform both movements.

At the moment a modification of the current design is under examination, to allow for the scanning-angle dependency of the waveguide length [7]. The effective length of the waveguide can be defined as the length from the crossing point of the beam with the axis of the waveguide to the end of the waveguide. There is a mechanism for changing the effective length while steering. By altering the CAM profile of the steerable mirror unit somewhat, the location of the crossing point between the beam and the waveguide axis can be changed. With this flexibility a profile can be designed that always ensures the optimal length of the waveguide for all steering angles.

\section{Front mirrors}

The mirrors in the front shield direct the beam under the required angles $\alpha$ and $\beta$ into the plasma. The mirrors will be focusing in both beam planes. The mirrors are elongated in the beam plane to accommodate the steering range of \pm 12 degrees from the square waveguide, see fig. 4. Active cooling is foreseen for these mirrors, and they will be bolted or welded to the port plug structure. Focusing is optimized in the plane orthogonal to the beam plane for a spot size 2.1 meters from the mirror. Focusing in the beam steering plane is more difficult because the incident beam from the square waveguide moves over the mirror surface. If there is focusing in the beam steering plane this reduces the effective steering range. Therefore focusing of the beam is a tradeoff between steering range and spot size [7].

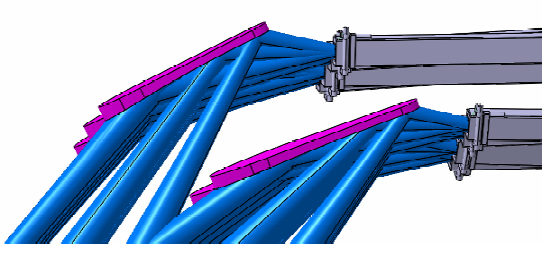

Figure 4: Mirror configuration

\section{Beam parameters}

Now the model includes a spherical mirror, instead of the flat one in previous versions. For each mirror, apart from the nominal beam (at $0^{\circ}$ steering), also a beam for the $\mathrm{q}=2$ surface and one for the $\mathrm{q}=3 / 2$ surface are given. This results in (much) smaller beams for $\mathrm{q}=2$ and (a bit) larger for $\mathrm{q}=3 / 2$.

Upper row, steering plane: Focusing in steering plane, nominal parameters, din = $300 \mathrm{~mm}, f=700 \mathrm{~mm}$, this results in a waist $w_{0}$ of $17.98 \mathrm{~mm}$, the divergence angle is 1.79 degrees, see fig.5. Left columns: nominal; middle: $\mathrm{q}=2$; right: $\mathrm{q}=3 / 2$.

\begin{tabular}{|l|l|l|l|l|l|l|}
\hline & $\mathrm{w}(\mathrm{mm})$ & $\mathrm{d}(\mathrm{mm})$ & $\mathrm{w}(\mathrm{mm})$ & $\mathrm{d}(\mathrm{mm})$ & $\mathrm{w}(\mathrm{mm})$ & $\mathrm{d}(\mathrm{mm})$ \\
\hline Mirror & 18.37 & -121.9 & 21.13 & 12.14 & 16.51 & -128.56 \\
\hline Plasma & 71.7 & -2222 & 58.56 & -2088 & 79.08 & -2229 \\
\hline
\end{tabular}

Lower row, steering plane: Focusing in steering plane, din $=250 \mathrm{~mm}, f=1125$ $\mathrm{mm}$, this results in a waist $w_{0}$ of $15.36 \mathrm{~mm}$, the divergence angle is 2.09 degrees Left columns: nominal; middle: $\mathrm{q}=2$; right: $\mathrm{q}=3 / 2$.

\begin{tabular}{|l|l|l|l|l|l|l|}
\hline & $\mathrm{w}(\mathrm{mm})$ & $\mathrm{d}(\mathrm{mm})$ & $\mathrm{w}(\mathrm{mm})$ & $\mathrm{d}(\mathrm{mm})$ & $\mathrm{w}(\mathrm{mm})$ & $\mathrm{d}(\mathrm{mm})$ \\
\hline Mirror & 16.81 & -187.0 & 19.38 & -245.64 & 15.41 & -148.89 \\
\hline Plasma & 85.0 & -2287 & 74.85 & -2346 & 89.50 & -2249 \\
\hline
\end{tabular}

\begin{tabular}{llll}
\hline Ec-13 6/28/2004 & Toon Verhoeven & Page 2 of 3
\end{tabular} 


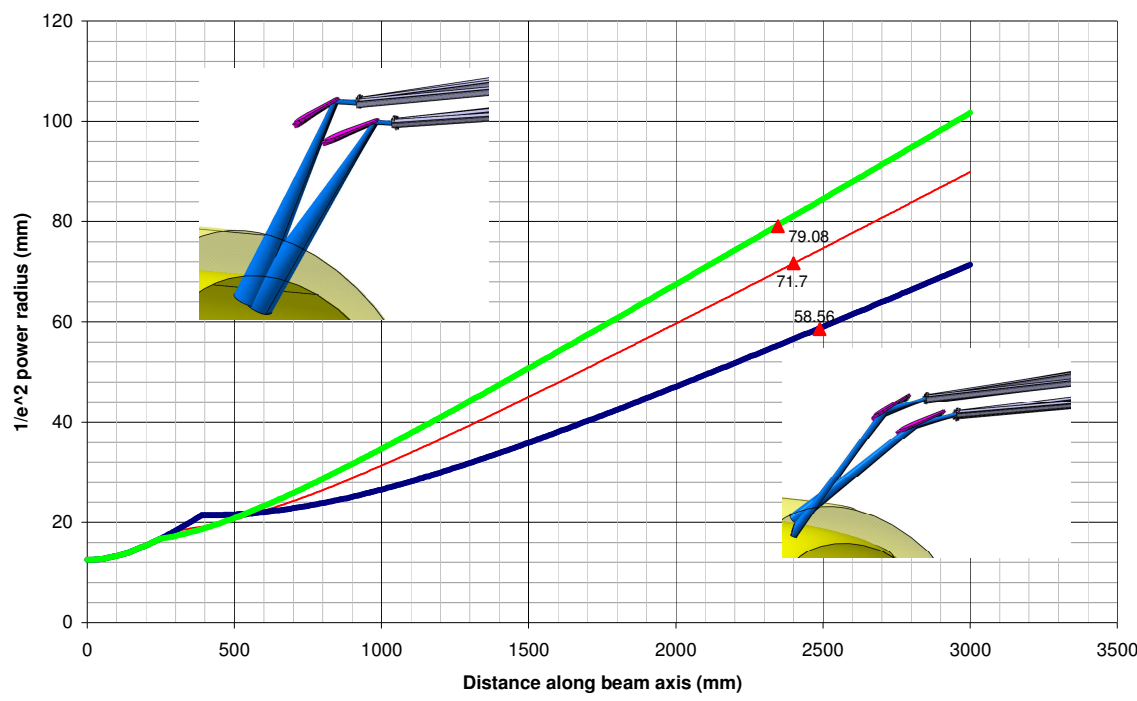

Figure 5: $\quad$ Beam envelope for the upper row showing $q=2$ (blue), nominal (red), and $q=3 / 2$ (green). Shown are the configurations for $q=3 / 2$ (upper plot) and $\mathrm{q}=2$ (lower)

\section{Definitions of parameters:}

$w=\quad$ Gaussian beam radius, $1 / \mathrm{e}$ of the E-field or $1 /\left(\mathrm{e}^{\wedge} 2\right)$ of the power.

div $=$ divergence of the Gaussian beam (far field angle).

$w_{0}=$ Gaussian beam waist

$d=\quad$ the location of the beam waist relative to the point where Rc and $\mathrm{w}$ are specified, positive is in the direction of the plasma.

\section{Two beams on one end mirror}

A further optimization is being studied at the moment. Two beams fall on 1 mirror, see fig.6. This enables to increase the distance between the square waveguide and the end-mirror, from 300 to $350 \mathrm{~mm}$ for the upper row and from 250 to $300 \mathrm{~mm}$ for the lower. This, in turn, allows having wider beams on the mirror and therefore this decreases the beam radii substantially at the relevant plasma surfaces. Furthermore, a lower peak power density on the mirror can be achieved, allowing for relaxed cooling requirements.

\section{Conclusions}

The design analyses so far have demonstrated the feasibility of the remote-steering approach in the ITER environment. In parallel to this work a detailed design of the mm-wave layout of the remote-steering concept for the upper-port launcher is underway to come to a consistent integration into the ITER environment. Furthermore, a full-scale mock-up line is being designed and build for testing at the appropriate ITER frequency and power level [9]. It is the intention to make the test mock-up very flexible in order to test all the options including upper and lower row mirror set-ups, multi-purpose and dedicated launchers. This work, supported by the European Communities under the contract of Association between EURATOM/FOM, was carried out within the framework of the European Fusion Programme with financial support from NWO. The views and opinions expressed herein do not necessarily reflect those of the European Commission. This work was carried out under the EFDA technology research programme activities, EFDA technology task TW3TPHE-ECHULA and B1.

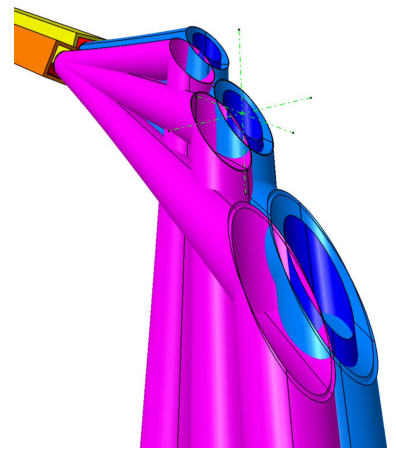

Figure 6: Two beams on one end mirror

\section{References}

[1] H Zohm, "THE CONCEPT OF ECRH/ECCD FOR ITER", this conf

[2] H Zohm, et al., "THE ITER ECRH UPPER LAUNCHER - PHYSICS GOALS AND DESIGN REQUIREMENTS", this conf

[3] S Nowak, et al., "ASTIGMATIC GAUSSIAN BEAMS IN PLASMAS", this conf

[4] G Ramponi, et al., "ITER ECRF UPPER LAUNCHER OPTIMISATION STUDIES", this conf

[5] F Volpe, et al., "FOKKER-PLANCK MODELLING OF ECCD FOR NTM STABILISATION IN ITER", this conf

[6] R Heidinger, et al., "DESIGN AND ANALYSIS OF WINDOWS AND STRUCTURAL COMPONENTS FOR THE ITER ECRH UPPER PORT PLUG", this conf

[7] A.G.A. Verhoeven, et al., "The ITER Remote-Steering ECW Upper-Port Launcher", The 28th Int. Conference on Infrared and MM Waves, Otsu, Japan, 29 Sep - 3 Oct, 2003, JSAP Catalog No. 031231 (2003), Editor: N. Hiromoto

[8] G. Gantenbein, et al., "HIGH-POWER TESTS OF A REMOTE STEERING LAUNCHER MOCK-UP AT $140 \mathrm{GHZ",} \mathrm{this} \mathrm{conf}$

[9] J.-P. Hogge, et al., "DEVELOPMENT OF A 2 MW, CW, 170 GHZ COAXIAL CAVITY GYROTRONS FOR ITER", this conf

\begin{tabular}{lll}
\hline Ec-13 6/28/2004 & Toon Verhoeven & Page 3 of 3
\end{tabular} 